\title{
Keuchhusten kommt zurück
}

- Eigentlich kann gegen die Durchimpfungsraten bei Pertussis nichts gesagt werden: In einer Untersuchung unter Schulanfängern in Bayern lag sie im Jahr 2008/2009 bei $92,2 \%$. Jedoch verschiebt sich die Krankheit immer mehr über die Jugendlichen und jungen Erwachsenen hin zu dem großen Pool der Erwachsenen. „Pertussis ist nicht nur eine Kinderkrankheit", bestätigte Prof. Dr. Johannes Liese aus Würzburg. Die KRESH-Studie ergab beispielsweise, dass bei rund $10 \%$ der Erwachsenen, die mehr als sieben Tage unter Husten litten, der Nachweis von Pertussis gelang. In Kalifornien, das ebenfalls gute Durchimpfungsraten aufweisen kann, kam es im letzten Jahr ebenfalls zu einem Anstieg der Erkrankung.
Plötzlich entsprach die Inzidenz wieder derjenigen aus den 50er-Jahren. Dabei starben zehn Säuglinge, die noch ungeimpft waren. „Pertussis kommt zurück“, war dazu der Kommentar von Liese.

Der Wiederanstieg hat mehrere Gründe: Zum einen schützt der azelluläre Impfstoff lediglich fünf bis maximal zehn Jahre. Jedoch nehmen Jugendliche die Auffrischimpfungen nicht genügend wahr. Auch Erwachsene sind häufig nicht vakziniert. Die aktuelle Empfehlung der STIKO lautet deshalb, Erwachsene mit dem Kombinationsimpfstoff Tetanus-Diphtherie-Pertussis (Tdap) zu impfen. Frauen mit Kinderwunsch sollten vor der Schwangerschaft ebenfalls eine Impfung erhalten. Liese appellierte auch an die
Chirurgen, im Falle einer Verletzung nicht nur die Tetanus-Spritze zu verabreichen, sondern gleich die gesamte Tdap-Impfung.

Die Hauptkrankheitslast tragen jedoch Säuglinge. Sie sollten deshalb schon mit zwei Monaten immunisiert werden. Weitere Impfungen folgen im Alter von drei, vier, elf bis 14 Monaten und fünf bis sechs Jahren. Die erste Impfung schützt nur teilweise. Erst die zweite Impfung bietet dem Säugling Protektion gegen Hospitalisationen. Zum Schluss gab Liese noch einen Tipp zur Diagnostik: Die PCR wird früh in der Erkrankung eingesetzt, bis maximal zwei Wochen nach Hustenbeginn. Ist ein Patient dagegen schon länger erkrankt, kommt die Serologie zum Einsatz.

nz

Liese J. Impfsymposium I: Pertussis

\section{Malaria: Expositionsprophylaxe ist das $\mathrm{A}$ und $\mathrm{O}$}

- Kinder, die an Malaria erkranken, können in drei Gruppen eingeteilt werden: zum einen Immigranten, zum anderen Kinder, bei denen mindestens ein Elternteil aus einem Malariaendemiegebiet stammt, und die sich bei einem Besuch in der elterlichen Heimat anstecken. Die dritte Gruppe sind deutsche Kinder bzw. Kaukasier, die sich während einer Reise oder nach einem Auslandsaufenthalt der Eltern im Endemiegebiet infizieren.

Prof. Dr. Ralf Bialek aus Kiel stellte Daten der ESPED-Studie vor. Dabei handelte es sich in den meisten Fällen um Malaria tropica, in der überwiegenden Zahl der Fälle wurden die Kinder in Westafrika angesteckt. Der Symptombeginn war meist innerhalb von vier Wochen nach der Reise zu beobachten. Fieber wurde bei $80 \%$ beobachtet, Allgemeinsymptome wie Müdigkeit und Abgeschlagenheit in $67 \%$ der Fälle. Weitere Symptome waren: Bauchschmerzen (34\%), Übelkeit/Erbrechen (33\%) und Schüttelfrost (24\%). Eine Hepato-/Splenomegalie war mit 23\% eher die Ausnahme, Kopfschmerzen eine Rarität. Die Kinder zeigten außerdem zum Teil schwere Anämien, auch Thrombozytopenien traten häufig auf.„Das C-reaktive Protein gibt jedoch keinen Hinweis auf eine Malariaerkrankung", erklärte Bialek.

Zur Expositionsprophylaxe gehört zunächst, das Endemiegebiet nicht zu bereisen, wenn die Ansteckungsgefahr besonders hoch ist. Wichtig ist auch das Tragen von langärme-

liger Kleidung. Imprägnierte Moskitonetze und Kleidung sind ebenfalls nützlich. Imprägnierte Moskitonetze können die Inzidenz einer unkomplizierten Malaria um 50\% reduzierten und die einer schweren Malaria um $45 \%$.

Repellents sind ein weiterer wichtiger Baustein der Expositionsprophylaxe: DEET (Diethyltoluamid) kann auch bei Kindern als 20\%ige Lösung angewendet werden. Die USamerikanischen Centers for Disease Control empfehlen sogar bis zu 50\%ige Lösungen. Icaridin (auch als Picaridin bekannt) kann ebenfalls bei Kindern eingesetzt werden, genauso wie $p$-Menthan-3,8-Diol (Citriodiol), der bisher wirksamste unter den pflanzlichen Wirkstoffen. Von anderen Repellents wie

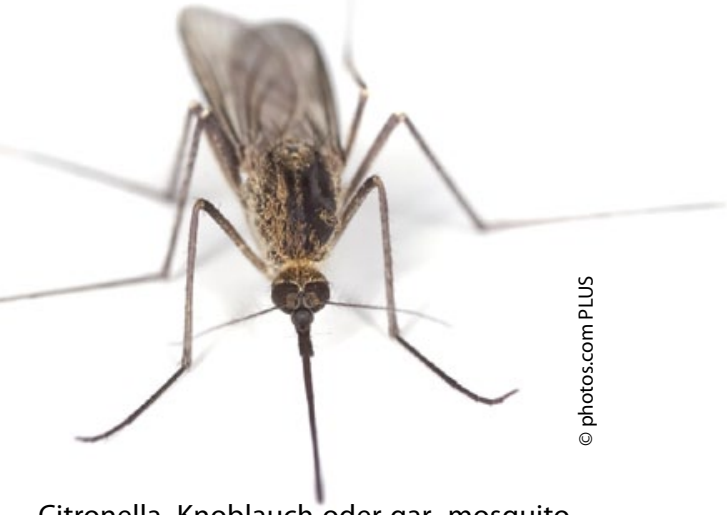

Citronella, Knoblauch oder gar "mosquito coils“ riet Bialek hingegen ab. Für die Räucherspiralen konnte sogar eine Assoziation zu Lungenkrebs hergestellt werden. Weitere Informationen sind bei der Deutschen Gesellschaft für Tropenmedizin unter www.dtg.org erhältlich.

$\mathrm{nz}$

Bialek R. Malaria bei Kindern

\section{Effektive Impfung gegen HPV}

— 480 Mädchen müssen gegen HPV geimpft werden, um einen Todesfall aufgrund eines Zervixkarzinoms zu verhindern. Um lediglich die Diagnose „Zervixkarzinom“ bei einer Frau zu vermeiden, sind 180 geimpfte Mädchen nötig. Für die Vermeidung einer hochgradigen squamösen intraepithelialen Läsionen (HSIL) sind es aber nur noch 24. Was allein schon die Diagnose HSIL bedeutet, schilderte Prof. Dr. Lutz Gissmann aus Heidelberg: psychische Belastung, operative Eingriffe mit dem Risiko für Frühgeburten, von den erheblichen Kosten für das Gesundheitssystem einmal ganz abgesehen. Bei anderen Erregern müssen im Vergleich dazu viel mehr Kinder geimpft werden, damit ein Todesfall vermieden werden kann: 40.000 bei Meningokokken C, 5.000-10.000 bei Streptococcus pneumoniae und 5.000 bei Haemophilus Influenzae B.

Gissmann L. Neue Entwicklungen zur HPVImpfung 